5th International Workshop on Astronomy and

Relativistic Astrophysics (IWARA2011)

International Journal of Modern Physics: Conference Series

Vol. 18 (2012) 1-9

(C) World Scientific Publishing Company

DOI: $10.1142 / \mathrm{S} 2010194512008112$

\title{
ENERGY RELEASE ASSOCIATED WITH QUARK PHASE TRANSITION IN NEUTRON STARS: COMPARATIVE ANALYSIS OF MAXWELL AND GLENDENNING SCENARIOS
}

\author{
ANI ALAVERDYAN, GRIGOR ALAVERDYAN ${ }^{\dagger}$ and SHUSHAN MELIKYAN \\ Faculty of RadioPhysics, Yerevan State University, \\ Manoogyan str. 1, Yerevan, 0025, Armenia \\ †galaverdyan@ysu.am
}

\begin{abstract}
We study the compact stars internal structure and observable characteristics alterations due to the quark deconfinement phase transition. To proceed with, we investigate the properties of isospin- asymmetric nuclear matter in the improved relativistic mean-field (RMF) theory, including a scalar-isovector $\delta$-meson effective field. In order to describe the quark phase, we use the improved version of the MIT bag model, in which the interactions between $u, d$ and $s$ quarks inside the bag are taken into account in the one-gluon exchange approximation. We compute the amount of energy released by the corequake for both cases of deconfinement phase transition scenarios, corresponding to the Maxwellian type ordinary first-order phase transition and the phase transition with formation of a mixed quark-hadron phase (Glendenning scenario).
\end{abstract}

Keywords: Neutron stars; quark phase transition; energy release.

PACS numbers: 97.60.Jd, 26.60.-c, 14.65.-q

\section{Introduction}

Neutron stars are objects with a very complicated, many-component and manylayered structure. The surface layer of such objects consists of an ordinary matter with atomic and molecular structure. In the inner layers of different depths, the conditions both for the rearrangement of the structural formations and for the creation of new constituents of matter are satisfied. In the central region of neutron star the density of matter reaches such high values that makes possible the appearance of various exotic particle species and phases such as hyperons, deconfined $u, d, s$ quarks and $\pi, K$ meson condensates. The existence of compact stars consisting of matter in a deconfined quark phase was predicted long ago ${ }^{1-3}$. Over the past few decades many researchers had been intensively studied various aspects related to the formation of exotic degrees of freedom in neutron stars and clarification of the observationally testing of dynamic processes, confirming the existence in the interiors of stars such constituents (for review see, e.g., Refs. 4, 5 and references therein). Phase transitions accompanied by discontinuities of the thermodynamic potentials 
are the most interesting because it leads to a dynamical rearrangement of neutron stars. Depending on the value of surface tension $\sigma_{s}$, the phase transition of nuclear matter into quark matter can occur in two scenarios ${ }^{6,7}$ : either ordinary first order phase transition at constant pressure with a density jump (Maxwell construction), or formation of mixed hadron-quark matter with a continuous variation of pressure and density (Glendenning construction) ${ }^{8}$. The question of whether the formation of a mixed phase is energetically favorable, given the finite dimensions of the quark structures inside nuclear matter, the Coulomb interaction, as well as the surface energy, has been examined in Refs. 7, 9, 10. It was shown that the mixed phase is energetically favorable for small values of the surface tension between the quark matter and the nuclear matter. Uncertainty of the surface tension values makes it impossible to determine the phase transition scenario which actually takes place. An important manifestation of the hadron-quark phase transition in the compact stars is a dynamical process of accretion of matter onto the surface of neutron stars with the hadron structure, which leads to fulfillment of conditions for the formation in the center of the star a new phase containing deconfined quarks. This type of transition can also occur in case of a rotating neutron star that is slowing down when the pressure in the center rises and exceeds the threshold value.

The process of catastrophic rearangement with formation of a quark core of finite radius at the star's center will be accompanied by release of a colossal amount of energy comparable to the energy release during a supernova explosion. These features of accreting neutron star near the critical configuration make it a potential candidate both for the gamma-ray bursts (GRBs) ${ }^{11,12}$ and for the gravitational wave $(\mathrm{GW})$ emission sources ${ }^{13,14}$. Note that a similar process of both restructuring and energy release takes place also in the case of pion condensation in the cores of neutron stars ${ }^{15-19}$.

Recent series of our articles ${ }^{20-24}$ were devoted to a detailed investigation of quark deconfinement phase transition of neutron star matter, when the nuclear matter is described in the relativistic mean-field (RMF) theory with the scalarisovector $\delta$-meson effective field. The calculation results of the mixed phase structure (Glendenning construction) are compared with the results of a usual first-order phase transition (Maxwell construction). This article is a continuation of this series. Here we aim to investigate the energy release and the change of integral parameters of compact stars due to the quark phase transition in the two alternative scenarios and to identify possible differences in the manifestations of this phenomenon.

\section{Model Equations of State for Compact Stars}

We use the EOS of Baym-Bethe-Pethick (BBP) ${ }^{25}$ for description of hadronic phase in the lower density region, corresponding to the outer and inner crust of the star. In nuclear and supranuclear density region $\left(n \geq 0.1 \mathrm{fm}^{-3}\right)$ was used the relativistic Lagrangian density of many-particle system consisting of nucleons, $p$, $n$, electrons and isoscalar-scalar $(\sigma)$, isoscalar-vector $(\omega)$, isovector-scalar $(\delta)$, and 
isovector-vector $(\rho)$ - exchanged mesons:

$$
\begin{array}{r}
\mathcal{L}=\bar{\psi}_{N}\left[\gamma^{\mu}\left(i \partial_{\mu}-g_{\omega} \omega_{\mu}-\frac{1}{2} g_{\rho} \vec{\tau}_{N} \vec{\rho}_{\mu}\right)-\left(m_{N}-g_{\sigma} \sigma-g_{\delta} \vec{\tau}{ }_{N} \vec{\delta}\right)\right] \psi_{N} \\
+\frac{1}{2}\left(\partial_{\mu} \sigma \partial^{\mu} \sigma-m_{\sigma} \sigma^{2}\right)-\frac{b}{3} m_{N}\left(g_{\sigma} \sigma\right)^{3}-\frac{c}{4}\left(g_{\sigma} \sigma\right)^{4} \\
+\frac{1}{2} m_{\omega}^{2} \omega^{\mu} \omega_{\mu}-\frac{1}{4} \Omega_{\mu \nu} \Omega^{\mu \nu}+\frac{1}{2} m_{\rho}^{2} \vec{\rho}^{\mu} \vec{\rho}_{\mu}-\frac{1}{4} \Re_{\mu \nu} \Re^{\mu \nu} \\
+\frac{1}{2}\left(\partial_{\mu} \vec{\delta} \partial^{\mu} \vec{\delta}-m_{\delta}^{2} \vec{\delta}^{2}\right)+\bar{\psi}_{e}\left(i \gamma^{\mu} \partial_{\mu}-m_{e}\right) \psi_{e}
\end{array}
$$

where $\sigma, \omega_{\mu}, \vec{\delta}$, and $\vec{\rho}^{\mu}$ are the fields of the $\sigma, \omega, \delta$, and $\rho$ exchange mesons, respectively, $m_{N}, m_{e}, m_{\sigma}, m_{\omega}, m_{\delta}, m_{\rho}$ are the masses of the free particles, $\psi_{N}=$ $\left(\begin{array}{l}\psi_{p} \\ \psi_{n}\end{array}\right)$ is the isospin doublet for nucleonic bispinors, and $\vec{\tau}$ are the isospin $2 \times 2$ Pauli matrices. Antisymmetric tensors of the vector fields $\omega_{\mu}$ and $\vec{\rho}_{\mu}$ given by

$$
\Omega_{\mu \nu}=\partial_{\mu} \omega_{\nu}-\partial_{\nu} \omega_{\mu}, \quad \Re_{\mu \nu}=\partial_{\mu} \vec{\rho}_{\nu}-\partial_{\nu} \vec{\rho}_{\mu}
$$

In our calculations we take $a_{\delta}=\left(g_{\delta} / m_{\delta}\right)^{2}=2.5 \mathrm{fm}^{2}$ for the $\delta$ coupling constant, as in Ref. 26. Also we use $m_{N}=938.93 \mathrm{MeV}$ for the bare nucleon mass, $m_{N}^{*}=$ $0.78 m_{N}$ for the nucleon effective mass, $n_{0}=0.153 \mathrm{fm}^{-3}$ for the baryon number density at saturation, $f_{0}=-16.3 \mathrm{MeV}$ for the binding energy per baryon, $K=300$ $\mathrm{MeV}$ for the incompressibility modulus, and $E_{\text {sym }}^{(0)}=32.5 \mathrm{MeV}$ for the asymmetry energy. Then five other constants, $a_{i}=\left(g_{i} / m_{i}\right)^{2}(i=\sigma, \omega, \rho), b$ and $c$, can be numerically determined: $a_{\sigma}=\left(g_{\sigma} / m_{\sigma}\right)^{2}=9.154 \mathrm{fm}^{2}, a_{\omega}=\left(g_{\omega} / m_{\omega}\right)^{2}=4.828 \mathrm{fm}^{2}$, $a_{\rho}=\left(g_{\rho} / m_{\rho}\right)^{2}=13.621 \mathrm{fm}^{2}, b=1.654 \cdot 10^{-2} \mathrm{fm}^{-1}, c=1.319 \cdot 10^{-2}$. The knowledge of the model parameters makes it possible to solve the set of four equations in a self-consistent way and to determine the re-denoted mean-fields, $\sigma \equiv g_{\sigma} \bar{\sigma}, \omega \equiv$ $g_{\omega} \bar{\omega}_{0}, \delta \equiv g_{\delta} \bar{\delta}^{(3)}$, and $\rho \equiv g_{\rho}{\overline{\rho_{0}}}^{(3)}$, which depend on baryon number density $n$ and asymmetry parameter $\alpha=\left(n_{n}-n_{p}\right) / n$. The standard QHD procedure allows to obtain expressions for energy density $\varepsilon(n, \alpha)$ and pressure $P(n, \alpha)$ (for details see Ref. 20).

To describe the quark phase an improved version of the MIT bag model was used, in which the interactions between $u, d, s$ quarks inside the bag are taken in a one-gluon exchange approximation ${ }^{27}$. We choose $m_{u}=5 \mathrm{MeV}, m_{d}=7 \mathrm{MeV}$ and $m_{s}=150 \mathrm{MeV}$ for quark masses, $B=60 \mathrm{MeV} / \mathrm{fm}^{3}$ for bag parameter and $\alpha_{s}=0.5$ for the strong interaction constant.

Using these equations of state for both the nucleonic and the quark phase, we can calculate the physical parameters of the phase transition as in case of Glendenning construction where these phases satisfy the Gibbs condition, are electrically charged separately, but lead to the global electrical neutrality of the system, as well as in the case when both phases are separately neutral and transition is a usual first-order phase transition corresponding to the well-known Maxwell construction. Model EOSs of neutron star matter for Glendenning and Maxwell construction 


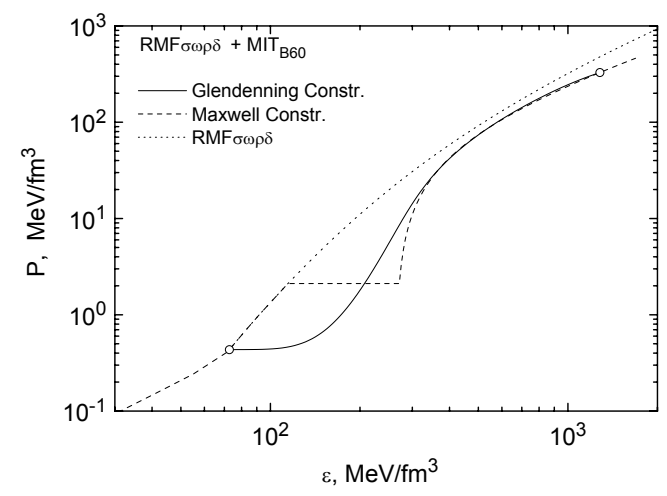

Fig. 1. EOS of neutron star matter for the two different hadron-quark phase transition constructions. Solid and dashed lines correspond to the Glendenning and Maxwell constructions, respectively, and the dotted line to the pure npe matter. Open circles represent the mixed phase boundaries.

cases are presented in Fig. 1. In case of the Maxwell construction the phase transition occurs at constant pressure $P_{0}=2.11 \mathrm{MeV} / \mathrm{fm}^{3}$ and nucleonic matter of energy density $\varepsilon_{N}=114.5 \mathrm{MeV} / \mathrm{fm}^{3}$ coexisted with the quark matter of energy density $\varepsilon_{Q}=271.4 \mathrm{MeV} / \mathrm{fm}^{3}$. In Glendenning construction case the deconfinement phase transition proceed through formation of a mixed hadron-quark phase. Boundaries of the mixed phase are $\varepsilon_{N}=72.79 \mathrm{MeV} / \mathrm{fm}^{3}, P_{N}=0.43 \mathrm{MeV} / \mathrm{fm}^{3}$ and $\varepsilon_{Q}=1280.88 \mathrm{MeV} / \mathrm{fm}^{3}, P_{Q}=327.75 \mathrm{MeV} / \mathrm{fm}^{3}$.

It was shown ${ }^{28}$ that for an ordinary first order phase transition, the density discontinuity parameter $\lambda=\varepsilon_{S} /\left(\varepsilon_{N}+P_{0}\right)$ plays a decisive in the stability of neutron stars with arbitrarily small cores made of the denser-phase matter. Here $P_{0}$ is coexistence pressure of two phases, and $\varepsilon_{N}, \varepsilon_{S}$ are the energy density of normal and superdense phases, respectively. Paraphrasing the conclusions of that paper, in case of a hadron-quark first order phase transition, we get the following stability criterions: if $\lambda<3 / 2$, then the neutron star with an arbitrarily small core of strange quark matter is stable, and if $\lambda>3 / 2$, neutron stars with small quark cores are unstable. In the latter case, for a stable star there is a nonzero minimum value for the radius of the quark core. Note that in the Maxwell construction case of deconfinement phase transition discussed in this article the value of jump parameter is $\lambda=2.327$.

\section{Changes in the Stellar Parameters and Energy Release due to Quark Phase Transition}

Using the neutron star matter EOSs obtained in previous section, we have integrated the Tolman-Oppenheimer-Volkoff (TOV) equations ${ }^{29,30}$ and obtained the 


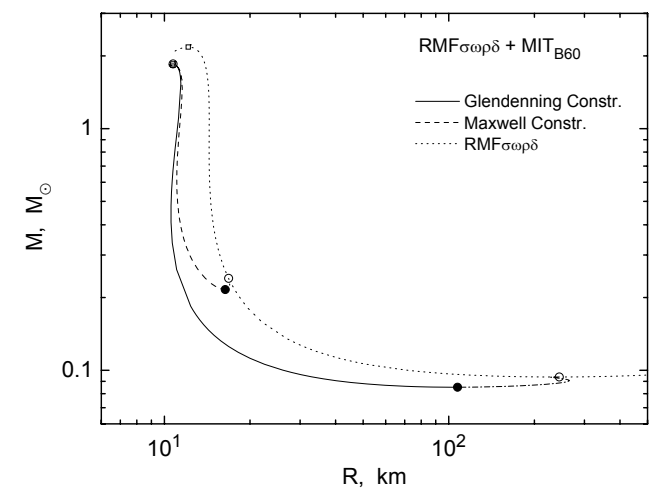

Fig. 2. Mass-radius relations for model EOSs of neutron star matter presented in Fig. 1. The meaning of the curves is the same as in Fig. 1. Open circles denote the critical configurations, solid circles denote the stable hybrid stars with minimal mass. Dash-dotted line between circles denote unstable stars branch.

gravitational mass $M$, radius $R$ and baryonic mass $M_{0}=m_{N} N_{B}\left(m_{N}\right.$ is the nucleon mass and $N_{B}$ the total number of baryons) of compact stars for the different values of central pressure $P_{c}$.

In Fig. 2 we show the $M(R)$ dependence of compact stars for different quark deconfinement phase transition scenarios. Open circles denote the critical configurations while solid circles denote the stable stars with minimal mass in the center of which there are deconfined quarks. Moreover, in the case of Maxwell construction this core is consisted of a quark-electron plasma, while in the Glendening construction case it is consisted of a mixed quark-hadron matter. We can see that in both cases of phase transition scenarios considered here there are unstable star branches between critical and minimum-mass configurations (dot-dashed line segment). The fact that in the case of Maxwell construction the $M-R$ relation has such a behavior is not surprising, since according to the Seidov criterium ${ }^{28}$ the infinitesimal core of denser matter in ordinary first order phase transition is unstable when $\lambda>3 / 2$. The corresponding EOS of neutron star matter considered here satisfies this condition. With regard to the case of Glendenning construction, the appearance of the unstable branch of compact stars with infinitesimal core consisting of a mixed hadron-quark matter, is not the standard case. The fact that in this scenario, the energy density is a continuous function of pressure and in most cases leads to a monotonic increase of stars mass at the lower boundary of the mixed phase, means that the configurations with an infinitesimal core of the mixed phase in many cases are stable. The case of the equation of state with a mixed phase, leading to a similar behavior of the stellar mass as a function of the central pressure was examined in the article $\mathrm{e}^{31}$. In contrast to our case, in this article the unstable branch of compact stars appears near the upper threshold of the mixed phase and catastrophic 
transition from hybrid star with a smaller core of mixed phase to the hybrid star with a large core of the mixed phase is realized. In case of Glendenning construction considered here the branch of unstable compact stars appear near the lower threshold of the mixed phase. In this case the accretion of matter onto the surface of an ordinary neutron star located below the critical configuration, the baryonic mass of the star increases, the star is reached a critical configuration and the transition to the configuration with deconfined quark phase is takes place.

It is worth noting that the maximum mass of stars, containing deconfined quarks is $M_{\max }=1.853 M_{\odot}$ for the case of Glendenning construction and $M_{\max }=1.828 M_{\odot}$ for the case of Maxwell construction.

Since the transition of ordinary neutron star to a star containing quark matter occurs at a constant baryon number, then the characteristics of the star is conveniently to represent as functions of baryonic mass $M_{0}$. The binding energy $E_{b i n d}=\left(M-M_{0}\right) c^{2}$ of compact stars as a function of baryonic mass $M_{0}$ for Maxwell and Glendenning hadron-quark phase transition scenarios is shown in Fig. 3.

Accretion of matter onto the surface of the critical configuration $C_{N}$ will lead to a jumpwise transition to the configuration $C_{Q}$, having a finite-size core consisting the deconfined quark matter. This transition will be accompanied by an enormous release of energy determined by the difference in binding energies of these configurations:

$$
E_{\text {release }}=E_{\text {bind }}\left(C_{Q}\right)-E_{\text {bind }}\left(C_{N}\right)=\left(M\left(C_{N}\right)-M\left(C_{Q}\right)\right) c^{2} .
$$

In Fig. 4 we plot the total energy release as a function of baryonic mass of star. One can see that in both cases of phase transition constructions the released energy increases with the increase of baryonic mass $M_{0}$. For a fixed value of the baryonic

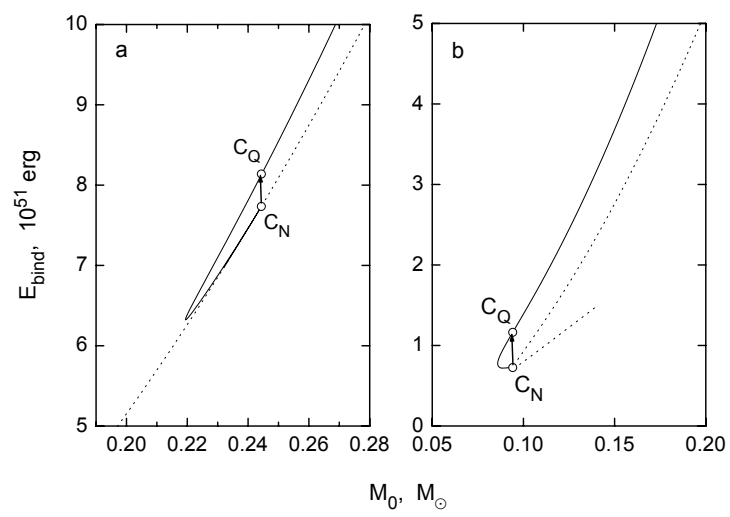

Fig. 3. Binding energy of compact stars as a function of baryonic mass $M_{0}$ for Maxwell (left panel) and Glendenning (right panel) constructions. Dotted line corresponds to the pure nuclear matter. $C_{N}$ denotes the critical configuration of hadronic star, and $C_{Q}$ presents the neutron star with same baryonic mass containing quark phase. 


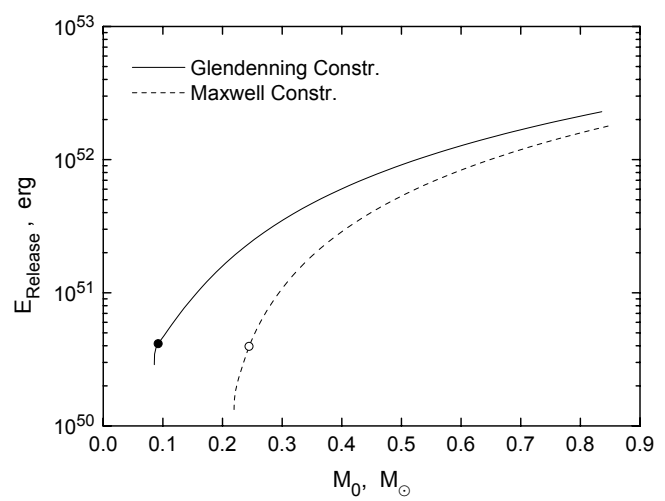

Fig. 4. The total energy release due to the hadron-quark phase transition as a function of baryonic mass of neutron star $M_{0}$ for Maxwell and Glendenning constructions. Circles correspond to the $C_{N} \rightarrow C_{Q}$ transition presented in Fig. 3 .

mass $M_{0}$ of star the conversion energy in the case of Glendenning construction more than in the case of Maxwell construction. In addition, the minimum required baryonic mass for the catastrophic rearrangement of the neutron star and the formation of a quark core in the center of the star greater in the case of the Maxwell construction compared to the Glendenning construction case. In the case considered here, the quark deconfinement phase transition in the neutron star interior leads to the energy release of the order $10^{50} \div 10^{52} \mathrm{erg}$.

Fig. 5 shows the changes of stellar radius $\Delta R=R_{Q}-R_{N}$ due to the quark deconfinement phase transition as a function of baryonic mass $M_{0}$ of a compact

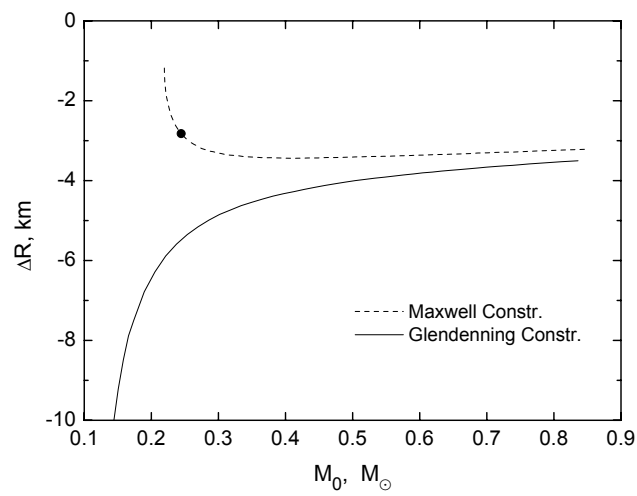

Fig. 5. Changes in the stellar radius due to the hadron-quark phase transition as a function of baryonic mass $M_{0}$ of a neutron star for Maxwell and Glendenning scenarios. 
star in both Glendenning and Maxwell construction cases. It is seen that in both cases the compact stars radii decrease. In addition, the behavior of changes $\Delta R$ for the two alternative scenarios are strongly distinguished.

\section{Conclusion}

In this article we have studied the changes of the internal structure and observable characteristics of the near-critical configurations of compact stars and associated energy release due to the quark deconfinement phase transition. For description of isospin-asymmetric nuclear matter we use the RMF theory, including a scalar-isovector $\delta$-meson effective field. The quark phase is described in frame of the improved version of the MIT bag model. Using the obtained characteristics of hadronic and strange quark matter phases, we calculate the neutron star matter EOS with quark-deconfinement phase transitions, corresponding to the Maxwell and Glendenning scenarios.

We find the dependence of conversion energy on the baryonic mass of neutron stars and analyze the changes in stellar radiuses due to the deconfinement phase transition.

We show that for a fixed value of the baryonic mass $M_{0}$ of star the conversion energy in the case of Glendenning construction more than in the case of Maxwell construction. The minimum required baryonic mass for the catastrophic rearrangement of the neutron star and the formation of a quark core in the center of the star greater in the case of the Maxwell construction compared to the Glendenning construction case. In the case considered here, the quark deconfinement phase transition in the neutron star interior leads to the energy release of the order $10^{50} \div 10^{52} \mathrm{erg}$.

Our obtained results will give the opportunity to clarify the observational differences between the two scenarios of quark-deconfinement phase transition and to formulate a specific test for determining the phase transition scenario taking place in reality.

\section{References}

1. D. D. Ivanenko, D. F. Kurdgelaidze, Astrofizika, 1, 479 (1965).

2. N. Itoh, Progress of Theoretical Physics, 44, 291 (1970).

3. R. Bodmer, Phys. Rev. D 4, 1601 (1971).

4. N. K. Glendenning, Compact Stars. Nuclear Physics, Particle Physics, and General Relativity, (Springer, Berlin, 2000).

5. P.Haensel, A. Y. Potekhin, D. G. Yakovlev Neutron Stars 1. Equation of State and Structure, (Springer, New-York 2007).

6. H. Heiselberg, C. J.Pethick, E. F. Staubo, Phys. Rev. Lett., 70, 1355 (1993).

7. H. Heiselberg, M. Hjorth-Jensen, Phys. Rep., 328, 237 (2000).

8. N. K. Glendenning, Phys. Rev. D 46, 1274 (1992).

9. D. N. Voskresensky, M. Yasuhira, T. Tatsumi, Nucl. Phys. A 723, 291 (2003).

10. T. Maruyama, S. Chiba, H-J. Shultze , T. Tatsumi, arXiv: 0708.3277 v1 [nucl.-th] (2007).

11. I. Bombaci, B. Datta, Astrophys. J.,530, L69-L72 (2000). 
12. A. Drago, G. Pagliara, J. Schaffner-Bielich, J. Phys. G: Nucl. Part. Phys.,35 , 014052 (2008).

13. G. F. Maranghello, C. A. Z. Vasconcellos, J. A. de Freitas Pacheco, Phys. Rev.D 66, 064027 (2002).

14. W. Lin, B. A. Li, J. Xu, C. M. Ko, D. H. Wen, Phys. Rev.D 83, 045802 (2011).

15. A. B. Migdal, A. I. Chernoutsan, I. N. Mishustin, Phys. Lett. 83B, 158 (1979).

16. P. Haensel, M. Proszynski, Astrophys. J., 258, 306 (1982).

17. J. L. Zdunik, P. Haensel, R. Schaeffer, Astron.\& Astrophys., 172, 95 (1987).

18. G. B. Alaverdyan, Yu. L. Vartanyan, Rep. Acad. of Sci. of Armenia, Physics, 88, 18 (1989).

19. Z. Berezhiani, I. Bombaci, A. Drago, F. Frontera, A. Lavagno, Astrophys. J., 568, 1250 (2003).

20. G. B. Alaverdyan, Astrophysics, 52, 132 (2009).

21. G. B. Alaverdyan, Gravitation \& Cosmology, 15, 5 (2009).

22. G. B. Alaverdyan, Natural Science, 2, 489 (2010).

23. G. B. Alaverdyan, Research in Astron. Astrophys., 10, 1255 (2010).

24. A. G. Alaverdyan, G. B. Alaverdyan, A. O. Chiladze, Int. Journal of Modern Phys. D 19, 1557 (2010).

25. G. Baym, H. Bethe, Ch. Pethick, Nucl.Phys.,A 175, 255, 1971.

26. B. Liu, V. Greco, V. Baran, M. Colonna, M. Di Toro, Phys. Rev. C 65, 045201 (2002).

27. E. Farhi, R. L. Jaffe, Phys. Rev.D 30, 2379 (1984).

28. H. Seidov, Astron. Zh., 48, 443 (1971).

29. R. Tolman, Phys. Rev. 55, 364 (1939).

30. J. Oppenheimer, G. Volkoff, Phys. Rev.55, 374 (1939).

31. I. N. Mishustin, M. Hanauske, A. Battacharyya, L. M. Satarov, H. Stocker, W. Greiner, Phys. Lett. B 552,1(2003). 